\title{
Adverse Driving Conditions Alert: Investigations on the SWIR Bandwidth for Road Status Monitoring
}

\author{
Massimo Bertozzi $^{1}$, Rean Isabella Fedriga ${ }^{1}$, and Carlo D’ Ambrosio ${ }^{2}$ \\ 1 Dip. Ing Informazione, Parco Area delle Scienze 181A, 43124 Parma, Italy \\ www.vislab.it \\ 2 Centro Ricerche FIAT, Strada Torino 50, 10043 Orbassano (TO), Italy \\ wWw. crf.it
}

\begin{abstract}
The 2WIDE_SENSE (WIDE spectral band \& WIDE dynamics multifunctional imaging SENSor Enabling safer car transportation) EU funded project is aimed at the development of a low-cost camera sensor for automotive applications able to acquire the full visible to Short Wave InfraRed (SWIR) spectrum, from 400 to $1700 \mathrm{~nm}$.

This paper presents the results obtained using this extended spectral responsivity sensor for a Road Status Monitoring application to inspect the vehicle's frontal area and detect layers of ice or water on the road surface.
\end{abstract}

Keywords: SWIR, road status monitoring, large bandwidth cameras, icy road, wet road.

\section{Introduction}

Rain both reduces visibility and makes roadway surfaces dangerous. Wet brakes are less effective too. Snow and ice cause roads to become even more slippery, especially when the temperature is at or below freezing. Slush makes difficult to steer, hard packed snow increases the danger of skidding and black ice makes driving extremely dangerous. Stopping distances on slippery pavement are from two to ten times farther than on dry pavement so that for a vehicle travelling at $30 \mathrm{~km} / \mathrm{h}$ they can get up to $52 \mathrm{~m}$ on black ice. Moreover, usually, Anti Brake Systems (ABS) are tuned for the most slippery scenario and therefore less effective than they can be in normal situations. Therefore the detection of a general road status or of the presence of slippery spots in front of the vehicle can significantly improve driving safety. It can be noticed that in Europe (EU-18) around 3800 casualties are due to wet, icy or snowy situations [9].

Most of the proposed solutions to this problem are not based on a true prediction but are focused on the estimation of the road friction namely the monitoring of tyres slippering. These approaches are mainly based on the use of inertial sensors or GPS or on the monitoring of the tyres noise [2, 7, 10]. Conversely, different perception approaches have been proposed for a true prediction like the use of radars [15] or lasers [13]. The use of standard cameras have been proposed as well [3,8,11,14] exploiting the different polarization of the light reflected from the road surface.

Anyway, the most promising approach seems the analysis of the different spectral content of the light reflected from the asphalt in dry, wet, icy, or snow conditions [4].

A. Petrosino (Ed.): ICIAP 2013, Part I, LNCS 8156, pp. 592 6012013.

(C) Springer-Verlag Berlin Heidelberg 2013 


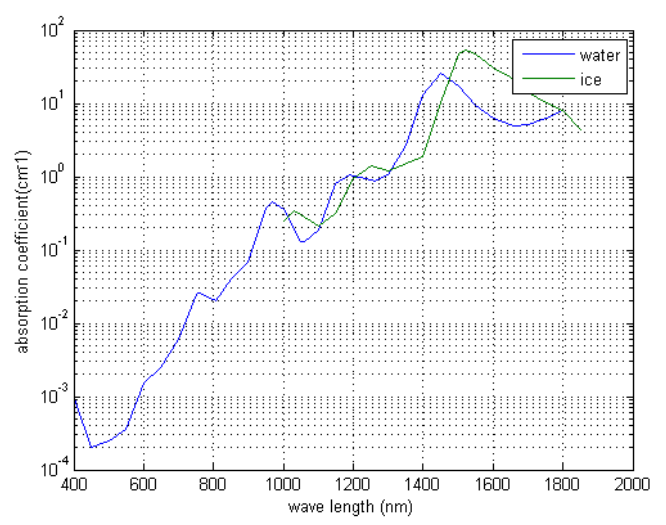

Fig. 1. Water and ice absorption coefficients

More precisely, the ShortWave InfraRed (SWIR, 0.9-1.7 $\mathrm{nm}$ ) bandwidth shows different light reflection patterns depending on the road status [5, 6, 12]. According to this result, some solutions based on the use of custom spectrometers have been already implemented, as examples, the Volvo's Road eye or the Vaisala's Road Weather Sensors family. While the use of a spectrometer can be effective, the proposed solutions are not suitable for on-board installation on vehicles for costs and size costraints. In addition, the monitored area is too small to allow an exhaustive mapping of slippery road areas when the vehicle is moving.

The European funded 2WIDE_SENSE collaborative project has the aim of developing a low-cost camera able to acquire in the full visible to SWIR bandwidth. This task required the development of a specific sensor and a large bandwidth lens. In addition, the camera will feature a filter on the sensors to enable the independent acquisition of 4 different spectral bandwidths. Each $2 \times 2$ pixels window on the sensor in fact will include 4 different pixel-level filters; namely the value of each pixel in the window will be due to a specific bandwidth the pattern of which will be specifically selected according to the automotive needs [1] also allowing to obtain other bandwidths of interest using differences amongst different pixels.

Tests discussed in this article were thus carried out employing a state-of-the-art InGaAs camera module in order to determine the most suitable bandwidths, from visible to SWIR, to recognize the road surface status. The prototypical camera module parameters have been explored (e.g. HDR, integration time, gain) by acquiring images of the asphalt in different conditions (dry, wet, icy, sunny, with shadows).

This paper is organized as follows: section 2 describes the hardware equipments employed. Section 3 describes the experimental measurements carried out both in lab and on-the-field in order to select the most appropriate wavelength bandwidths useful to detect non-dry road condition and to select the optical filter characteristics. Section 4 reports the results obtained and section 5 states our final considerations. 


\section{Hardware Equipment}

The multispectral camera module developed during the project has been available and therefore tested only during the final stage of the 2WIDE_SENSE experiments. A detailed description of the camera sensor, the filter pattern and the large bandwidth lens can be found in [1].

Conversely, most of the tests have been carried out using a state-of-the-art InGaAs camera module equipped with a SWIR high transmission lens. In order to mimic and evaluate the most suitable filters for the final prototype, a number of different filters has been used and tested (see fig. 2).

The camera used for tests is the OWL SW1.7 High sensitivity InGaAs FPA produced by Raptor Photonics and equipped with a sensor developed by Alcatel-Thales III-V Lab, both partners of the project consortium. The camera has a sensitivity bandwidth in the 400-1700 $\mathrm{nm}$ interval covering the whole spectrum from visible to the SWIR and acquires $320 \times 25614$ bit images within a $500 \mathrm{~ns}-500 \mathrm{~ms}$ exposure interval.

The lens used is the OB-SWIR25/2 developed and produced by Optec SpA. It is a high transmission lens featuring a transmission rate $>94 \%$ in the 900-1700 $\mathrm{nm}$ interval. The focal length is $25 \mathrm{~mm}$ with a $35.5 \mathrm{deg}$ angle of view.

In order to test a number of spectrum bandwidths and to compare the quantity of light reflected by the asphalt for different conditions and wavelengths, several filters have been used. In the preliminary phase of the project tuneable liquid crystal filters have been employed to perform several temporal sequential acquisitions. These tuneable filters allowed to choose different wavelengths with a $20 \mathrm{~nm}$ bandwith resolution from $850 \mathrm{~nm}$ to $1800 \mathrm{~nm}$ and a transmittance around $60 \%$. In the following phase a filter wheel (see fig. 2 b) with 12 filters has been installed between the lens and the camera allowing to select between the available filters. This is a manual operation and therefore limits the use of the filters to still objects.

\section{Indoor Tests}

The indoor acquisition sessions and measurements for this activity were performed at CRF (Centro Ricerche FIAT) in their electro-optics laboratory using the state-of-the-art InGaAs camera and a set of tunable liquid crystal filters as illustrated in section 2 .
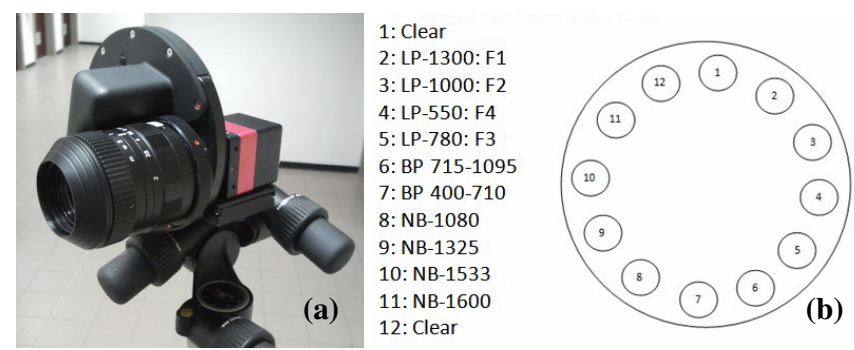

Fig. 2. The test setup: $(a)$ the camera and $(b)$ the 12 slots filter wheel outline 

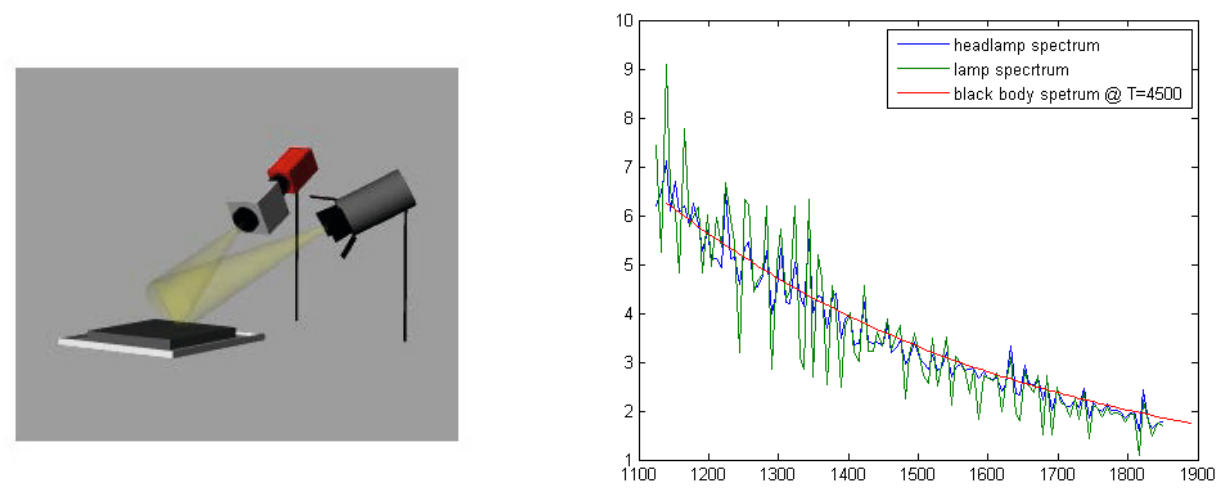

Fig. 3. Laboratory camera and lamp set-up and measures of lamp (green line) and headlamp (blue line) spectra compared to the black body (red line) one

Left image in fig. 3 outlines the laboratory set-up. Asphalt samples were collected and placed in a plastic box covered with commercial aluminum foil to prevent plastic's NIR absorption and avoid interferences in the acquired spectra. The asphalt surface was then illuminated with a stabilized $24 \mathrm{~V}$ halogen lamp and the images acquired by the state-of-the-art InGaAs camera described in section 2. The lamp spectrum has been analyzed by a calibrated spectrometer. Graph in fig. 3 reports the spectra comparison between the laboratory acquisition lamp (a vehicle's $\mathrm{H7}$ halogen headlamp) and the blackbody at $4500 \mathrm{~K}$.

Several tests have been done in order to set the most appropriate camera control parameters. The camera cooler has been enabled to stabilize the device at $25^{\circ} \mathrm{C}$ and the NUC (Non-Uniformity Correction) has been applied before each acquisition.

Asphalt samples have been modified in order to reproduce different road states: dry, damp, wet (less than $1 \mathrm{~cm}$ water thickness), very wet (about $1 \mathrm{~cm}$ water thickness), icy. For each configuration, different illumination conditions were analyzed, from full light to shadow, using metallic stops so as to minimize direct illumination without modifying the lamp spectrum.

\section{Outdoor Tests}

Outdoor tests have been performed using both the state-of-the-art InGaAs camera with the OB-SWIR25/2 lens and the filter wheel as shown in fig. 2]a.

The acquisition sessions for this activity were done at daytime with sunny and cloudy weather conditions and the road surface both dry and wet or iced in some areas as shown in the examples reported in fig. 4.

All combinations of gain and integration time values were also investigated to find the most suitable acquisition parameters for the RSM function. Some examples of these tests are shown in fig.5. 

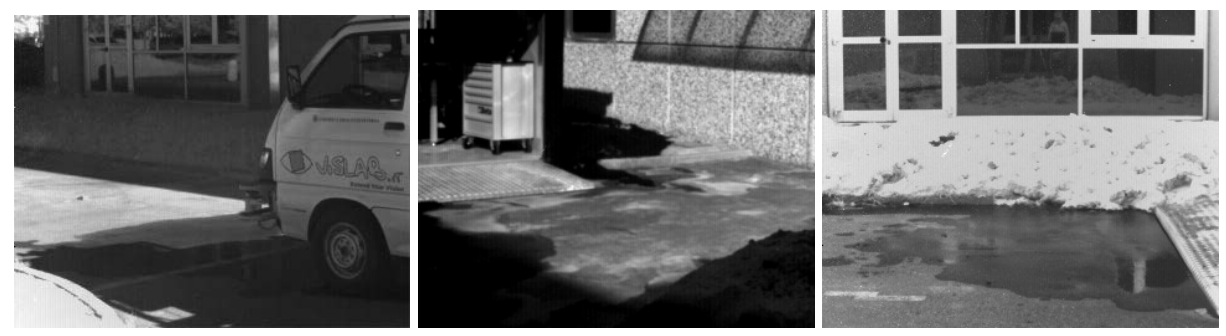

Fig. 4. Images acquired in the whole visible to SWIR bandwidth (no filters) with the state of the art camera. Different illumination and road surface conditions are shown.
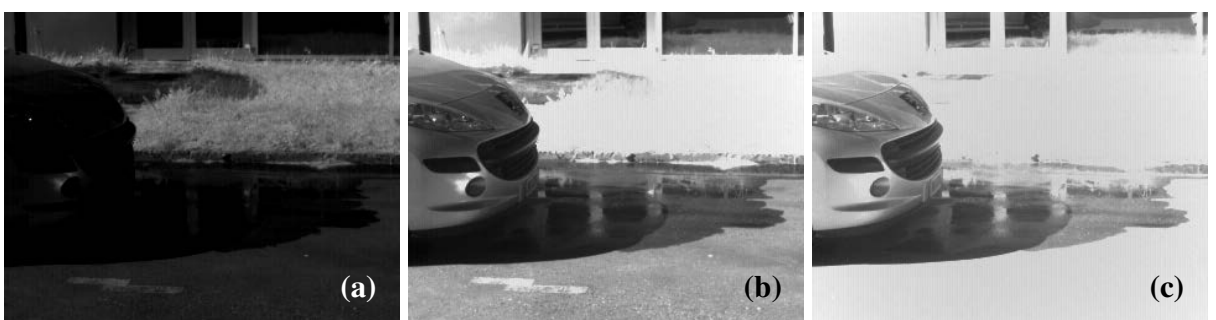

Fig. 5. Images acquired in the whole visible to SWIR bandwidth (no filters) with the state-of-theart camera. Low gain and three different integration times have been selected: $1 \mathrm{~ms}(\mathrm{a}), 5 \mathrm{~ms}(\mathrm{~b})$ and $10 \mathrm{~ms}(\mathrm{c})$.

\section{Indoor Results}

In fig. 6 the results of two different asphalt conditions under different illumination have been reported. 4 ROIS 1 were randomly chosen as test specimens on the asphalt surface (brighter areas of the images belong to the alumunium covered container). Fig. 6 right side reports the corresponding calculated SWIR spectra. For each condition, using the tuneable filters, several images of the samples have been acquired with $50 \mathrm{~nm}$ step from $850 \mathrm{~nm}$ to $1800 \mathrm{~nm}$. For each ROI, the mean pixel intensity has been computed then the mean intensity of the $1800 \mathrm{~nm}$ band subtracted. Since the imager sensitivity in $1800 \mathrm{~nm}$ spectral region is negligible, the measure could be used to calculate the pixel background values. This operation allowed to obtain intensity values less affected by noise: $I=\operatorname{mean}(I(R O I))-b k g$ where $\left.b k g=\operatorname{mean}\left(i m g_{1800}\right)\right)$.

ROI intensity values plotted as a function of wavelength are used to evaluate the spectrum corresponding to each sample in the considered condition. Some comments could be derived:

- the spectrum in dry conditions could be predicted by multiplying the lamp spectrum with the imager spectral responsivity and filter nominal transmittance; the asphalt spectral contribution is practically flat in the considered wavelength range

- there is a noticeable spectral modification from dry to wet conditions around the $1500 \mathrm{~nm}$ wavelength

\footnotetext{
${ }^{1}$ Regions Of Interest
} 

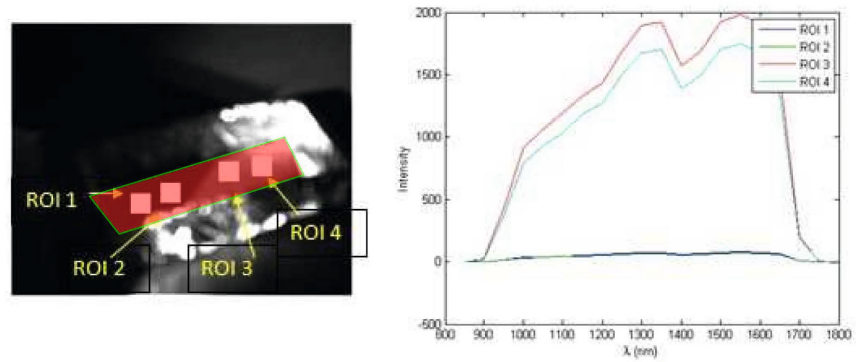

DRY
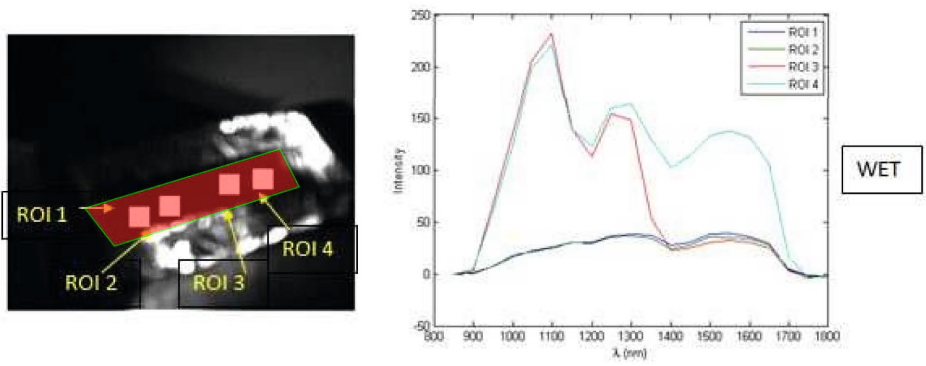

Fig. 6. Laboratory tests with the asphalt sample images (left) acquired in the halogen lamp's full bandwidth light conditions and the 4 ROIs mean pixel intensities graphs (right). The actual size of the asphalt sample is emphasized using the superimposed red bounding box.

\begin{tabular}{|c|c|c|c|c|c|c|}
\hline $\begin{array}{c}\text { Spectrometer } \\
\text { filters }\end{array}$ & $\begin{array}{c}\text { Asphalt } \\
\text { condition }\end{array}$ & $\mathbf{1 5 0 0 / \mathbf { 1 1 0 0 }}$ & $\mathbf{1 5 0 0 / \mathbf { 1 2 0 0 }}$ & $\mathbf{1 5 0 0 / \mathbf { 1 3 0 0 }}$ & $\mathbf{1 5 0 0 / \mathbf { 1 4 0 0 }}$ & $\mathbf{1 5 0 0 / \mathbf { 1 6 0 0 }}$ \\
\hline ROI_1 & $\mathrm{dry}$ & 1.57 & 1.30 & 1.01 & 1.23 & 1.02 \\
\hline ROI_2 & $\mathrm{dry}$ & 1.60 & 1.33 & 1.02 & 1.22 & 1.01 \\
\hline ROI_3 & $\mathrm{dry}$ & 1.63 & 1.36 & 1.02 & 1.23 & 1.00 \\
\hline ROI_4 & $\mathrm{dry}$ & 1.64 & 1.34 & 1.02 & 1.23 & 1.01 \\
\hline
\end{tabular}

\begin{tabular}{|c|c|c|c|c|c|c|}
\hline $\begin{array}{c}\text { Spectrometer } \\
\text { filters }\end{array}$ & $\begin{array}{c}\text { Asphalt } \\
\text { condition }\end{array}$ & $\mathbf{1 5 0 0 / \mathbf { 1 1 0 0 }}$ & $\mathbf{1 5 0 0 / \mathbf { 1 2 0 0 }}$ & $\mathbf{1 5 0 0 / \mathbf { 1 3 0 0 }}$ & $\mathbf{1 5 0 0 / 1 4 0 0}$ & $\mathbf{1 5 0 0 / 1 6 0 0}$ \\
\hline ROI_1 & wet & 0.53 & 0.69 & 0.53 & 1.27 & 1.00 \\
\hline ROI_2 & wet & 0.13 & 0.22 & 0.17 & 1.50 & 0.82 \\
\hline ROI_3 & wet & 0.23 & 0.43 & 0.33 & 1.35 & 0.99 \\
\hline ROI_4 & wet & 0.60 & 1.06 & 0.82 & 1.29 & 1.00 \\
\hline
\end{tabular}

Fig. 7. Ratios relative to fig. 6 asphalt samples 
- in non-illuminated ROIs the spectrum is flat and no significant modifications can be observed between the different asphalt conditions

In order to quantify the differences among spectra, these results have been analyzed considering the ratios between some wavelengths. The tables in fig. 7 illustrate the ratios corresponding to the analyzed ROIs. Columns with the 1500/1100 ratios showed the strongest changes in the diverse asphalt conditions.

The most relevant data from these analysis is show how the illumination can be an issue in developing an algorithm for the road monitoring.

\section{Outdoor Results}

The same methodology described in section 5 has been applied on images acquired in real scenarios. Dry, wet and icy road conditions at daytime have been investigated.

In the following, two scenes showing different illumination and road conditions have been selected (see fig. 8 and fig. 9). The spectral analysis has been done measuring the

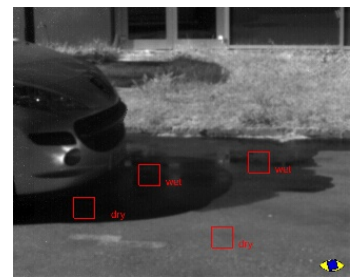

$L P 1300$

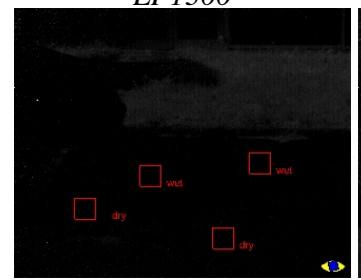

NB1533

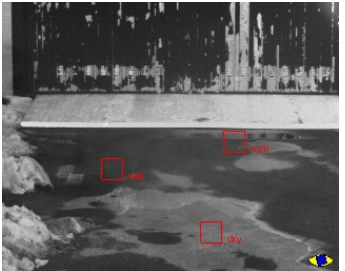

$L P 1000$

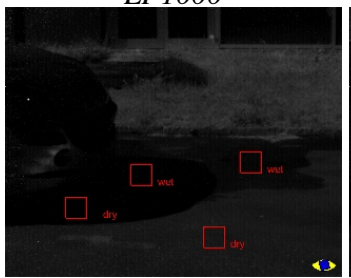

NB1600

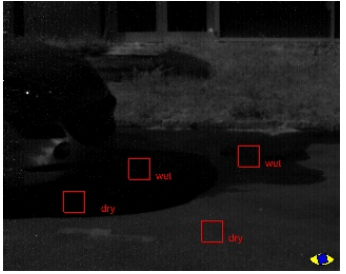

$N B 1325$

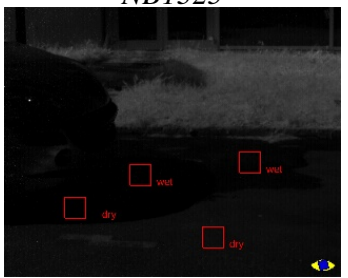

NB1080

\begin{tabular}{|c|c|c|c|c|c|c|}
\hline \multicolumn{7}{|c|}{ Exposure time $=10,0 \mathrm{~ms} / \mathrm{ROI}=20 \times 20$ pixel } \\
\hline $\begin{array}{l}\text { Filter wheel } \\
\text { filters }\end{array}$ & $\begin{array}{c}\text { Asphalt } \\
\text { condition }\end{array}$ & $\begin{array}{l}\text { NB1533/ } \\
\text { LP1000 }\end{array}$ & $\begin{array}{c}\text { NB1533/ } \\
\text { NB1080 }\end{array}$ & $\begin{array}{l}\text { NB1533/ } \\
\text { LP1300 }\end{array}$ & $\begin{array}{c}\text { NB1533/ } \\
\text { NB1325 }\end{array}$ & $\begin{array}{c}\text { NB1533/ } \\
\text { NB1600 }\end{array}$ \\
\hline ROI_1 & shadow - wet & 0.0186 & 0.1491 & 0.0470 & 0.2360 & 0.2119 \\
\hline ROI_2 & sun - wet & 0.0303 & 0.2014 & 0.0617 & 0.3193 & 0.2939 \\
\hline ROI_3 & shadow - dry & 0.0226 & 0.1755 & 0.0501 & 0.2844 & 0.2604 \\
\hline ROI_4 & sun - dry & 0.0216 & 0.1744 & 0.0486 & 0.2903 & 0.2641 \\
\hline
\end{tabular}

Fig. 8. Summer scene: dry and wet asphalt areas, illuminated and not, in a clear sky day. The table underneath shows the corresponding ratios. 


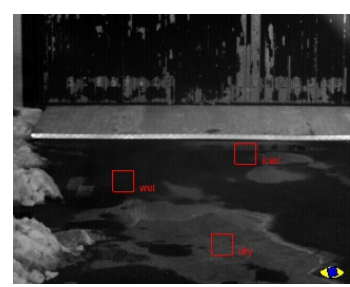

$L P 1300$

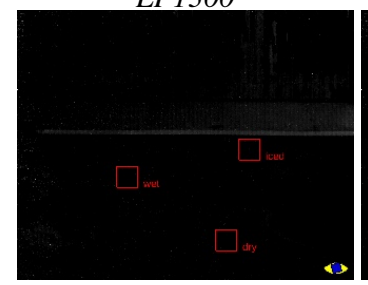

$N B 1533$

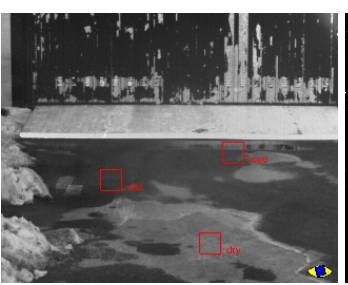

$L P 1000$

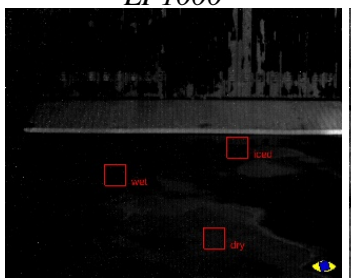

NB1600

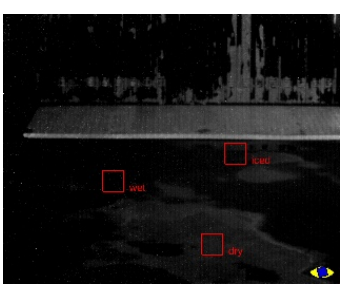

$N B 1325$

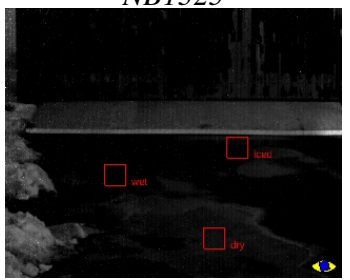

NB1080

\begin{tabular}{|c|c|c|c|c|c|c|}
\hline \multicolumn{7}{|c|}{ Exposure time $=30,980 \mathrm{~ms} \&$ ROI $=20 \times 20$ pixel } \\
\hline $\begin{array}{c}\text { Filter wheel } \\
\text { filters }\end{array}$ & $\begin{array}{c}\text { Asphalt } \\
\text { condition }\end{array}$ & $\begin{array}{c}\text { NB1533/ } \\
\text { LP1000 }\end{array}$ & $\begin{array}{c}\mathbf{N B 1 5 3 3 /} \\
\mathbf{N B 1 0 8 0}\end{array}$ & $\begin{array}{c}\mathbf{N B 1 5 3 3 /} \\
\text { LP1300 }\end{array}$ & $\begin{array}{c}\mathbf{N B 1 5 3 3 /} \\
\mathbf{N B 1 3 2 5}\end{array}$ & $\begin{array}{c}\text { NB1533/ } \\
\text { NB1600 }\end{array}$ \\
\hline ROI_1 & iced & 0.0030 & 0.1085 & 0.0102 & 0.0633 & 0.1284 \\
\hline ROI_2 & wet & 0.0065 & 0.1768 & 0.0208 & 0.1125 & 0.2108 \\
\hline ROI_3 & dry & 0.0036 & 0.1951 & 0.0117 & 0.0777 & 0.1240 \\
\hline
\end{tabular}

Fig. 9. Winter scene: dry, wet and icy asphalt areas in a cloudy day. The table underneath shows the corresponding ratios.

intensity values of the selected ROIs by using the filters included in the filter wheel operating in the SWIR bandwidth only.

The resulting ratios, shown in fig. 8 and fig. 9 , underline a behavior comparable to the indoor data although some relevant differences are noticeable:

- ratio values are different respect to lab ones due to the different source spectrum, halogen lamp in the lab and sun outdoor

- due to modification in illumination condition (clouds, etc.) during the acquisition (spectra are collected by means of temporal sequential measurements of the filter wheel filters), it is not possible to find a ratio as good indicator for road condition.

Taking into account the previous considerations, several measurements have been done in order to characterize how the presence of clouds could affect the ratios. Spectra in a changeable weather day, initially with clouds and then clear, have been collected using a calibrated spectrometer. In fig. 10, a the temporal spectral evolution is compared to the theoretical solar spectra at sea level (black line). 


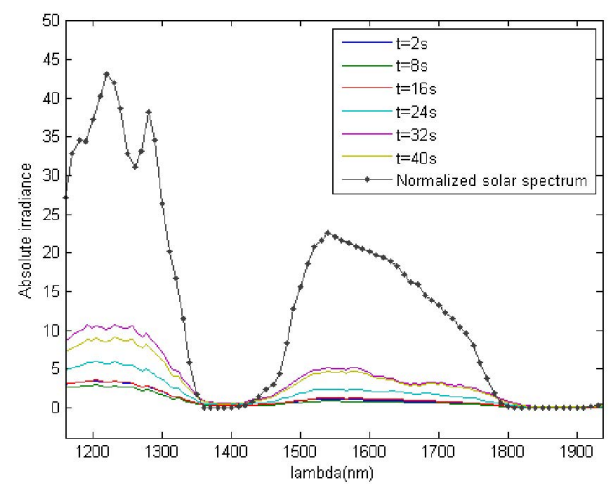

$a$

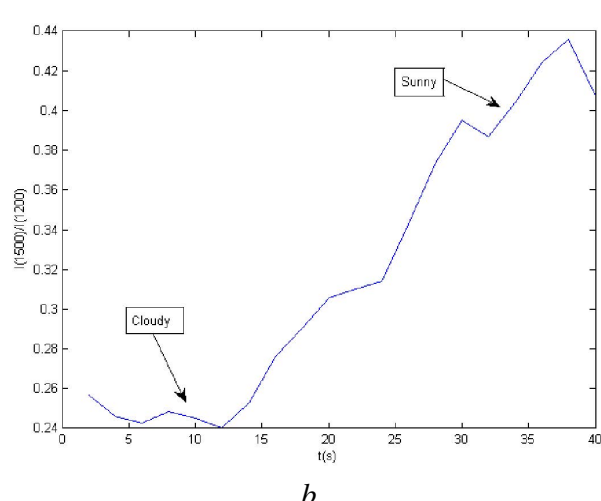

$b$

Fig. 10. Dealing with clouds: $(a)$ solar spectra collected during a cloudy day and $(b)$ variations of the $\mathrm{I}(1500) / \mathrm{I}(1200)$ ratio

In order to understand the contribution of the clouds, we collected some outdoor spectra during a cloudy day. In fig. 10, $b$ are shown the variations of the $\mathrm{I}(1500) / \mathrm{I}(1200)$ ratio during the acquisition. It has not been possible to evaluate the I(1500)/I(1100) ratio due to the spectral sensitivity limitation of the spectrometer.

From these measurements it can be noticed that illumination changes not only affect the intensity levels at all wavelengths but, due to the extra absorption of cloud water molecula, some wavelength ranges, i.e. $1500 \mathrm{~nm}$, are more deprived.

\section{Conclusions}

SWIR is nominally the wavelength range of about 1 to $2 \mathrm{~nm}$ that, similar to visible light, responds primarily to light reflected from objects rather than to thermal emissions coming from those objects. Therefore the only applications served by SWIR are those which benefit from the reduced scattering effects of longer wavelengths like illumination from invisible sources as passive illumination due to the upper atmosphere's night glow or active illumination coming from eye-safe lasers or from thermal emissions of objects with temperatures above $150^{\circ} \mathrm{C}$. So illumination is an important issue when dealing with SWIR images.

Our tests have shown that where for indoor acquisitions the lamp spectrum affects results only by a multiplicative factor, outdoor the unpredictable changes in illumination not only affect the intensity levels at all wavelengths but, due to the extra absorption of cloud water molecula, different wavelength ranges are also differently affected. Image processing techniques applied to satellite and airborne pictures have also been taken into account looking for a procedure to limit this unwanted behavior, but all spectral analysis techniques are applied to images clear of clouds, a hard restriction which is totally unsuitable for functions to be applied in the automotive field. 
Acknowledgments. The work described in this paper has been developed in the framework of the 2WIDE_SENSE (WIDE spectral band \& WIDE dynamics multifunctional imaging SENSor Enabling safer car transportation) Project funded by EU within the FP7 Seventh Framework Programme.

\section{References}

1. Bertozzi, M., Fedriga, R.I., Miron, A., Reverchon, J.-L.: Pedestrian Detection in Poor Visibility Conditions: Would SWIR Help? In: Procs. of the IAPR Intl. Conf. on Image Analysis and Processing, Naples, Italy (September 2013)

2. Bian, M., Li, K., Jin, D., Lian, X.: Road Condition Estimation for Automotive Anti-Skid Control System Based on BP Neural Network. In: Procs. of the IEEE Conf. on Mechatronics \& Automation, pp. 1017-1022. IEEE Computer Society, Niagara Falls (July 2005)

3. Casselgren, J., Jokela, M., Kutila, M.: Slippery road detection by using different methods of polarised light. In: Meyer, G. (ed.) Advanced Microsystems for Automotive Applications 2012, vol. 113, pp. 207-220. Springer, Heidelberg (2012)

4. Casselgren, J., Sjödahl, M., LeBlanc, J.P.: Angular spectral response from covered asphalt. Applied Optics 46(20), 4277-4288 (2007)

5. Casselgren, J., Sjödahl, M., LeBlanc, J.P.: Model-based winter road classification. International Journal of Vehicle Systems Modelling and Testing 7(3), 268-284 (2012)

6. Hansen, M.P., Malchow, D.S.: Overview of SWIR detectors, cameras, and applications. In: Procs. of the SPIE. Thermosense XXX, vol. 6939 (March 2008)

7. Akama, S.I., Tabaru, T., Shin, S.: Bayes Estimation of Road Surface Using Road Noise. In: Procs. of the IEEE Conf. of Industrial Electronics Society, pp. 2923-2928. IEEE Computer Society, Busan (November 2004)

8. Jokela, M., Kutila, M., Lu, L.: Road Condition Monitoring System Based on a Stereo Camera. In: Procs. of the IEEE Conf. on Intelligent Computer Communication and Processing, pp. 423-428 (August 2009)

9. KfV, NTUA, SWOV, and TRL. SafetyNet, Annual Statistical Report 2008. Technical report, European Road Safety Observatory (2008)

10. Lin, P.P., Ye, M., Lee, K.-M.: Intelligent Observer-Based Road Surface Condition Detection and Identification. In: Procs. of the Conf. Systems, Man and Cybernetics, pp. 2465-2470. IEEE Computer Society, Cleveland (October 2008)

11. Lu, Y., Higgins-Luthman, M.J.: Black ice detection and warning system. US Patent App. 11/948, 086 (November 30, 2007)

12. Malchow, D.: NIR Trends: Machine vision in the Short Wave Infrared. In: UTC Aerospace Systems (Sensors Unlimited Products). Goodrich Corporation (March 2009)

13. Mika, M., Hiroyuki, Y., Takao, K., Takeshi, I., Mitsuo, S.: Road Surface Condition Detector Using High Peak Power Fiber Laser. Transactions of the Institute of Electrical Engineers of Japan 10(4), 1198-1204 (2000)

14. Omer, R., Fu, L.: An automatic image recognition system for winter road surface condition classification. In: Procs. of the IEEE Intelligent Transportation Systems 2010, pp. 1375-1379 (September 2010)

15. Viikari, V., Varpula, T., Kantanen, M.: Automotive Radar Technology for Detecting Road Conditions. Backscattering Properties of Dry, Wet, and Icy Asphalt. In: Procs. of the 5th European Radar Conference, pp. 276-279. IEEE Computer Society, Amsterdam (2008) 